\title{
Sachregister zu Band 92
}

Die îett gedruckten Zahlen bedeuten Eigenarbeiten. Bb. = Buchbesprechungen.

A

Ablatio retinae, familiäre 264

- $\quad$ und Glaskörper 115

- spontane Anlegung einer - Ill

- $\quad$ Rezidiv nach erfolgreicher Operation der - 260

- durch Trauma 86Agranulozytose, Augenerkran-

kungen bei $\cdot-193$ Akkommodation 171

- $\quad$ Modell der - 122Akrothecium 60Aktinomyzes 60Akustisch-chromatische Synopsien 258 Amblyopie, Simulationsverhü-

tung bei der Sehschärfebestim-

mung bei - 119 Amöben 65 Aniridie, angeborene bei Mutter

und Kind 247 Anisometropie, Sehtäuschungen

bei der Korrektion von - 119 Ankylostomum 63 Antipyrin, Augenschädigung

durch - 78 Argyrose 242 Arsenvergiftung 76 Arteria centralis retinae,

Spasmen der - 126 Ascorbinsäure im Auge 98, 99 Aspergillus fumigatus 60 Aspirin,

Augenschädigung durch

- 78 A. T. 10 - 187 Atlas der Augenkrankheiten 372

(Bb.) Augapfel, nicht exulzerierte Skle-

rose der Bindehaut des - 300 Auge, Darstellung des - in der

expressionistischen Kunst 158 Augenkrankheiten, Atlas der -

$372(\mathrm{Bb}$.) Augenhintergrund, familiäre

hyaline Entartung des - 188

- grippöse Hämorrhagien am -207

Avitaminosen 237

B

Bacillus fusiformis 47

oedematis maligni 46

pyocyaneus 45

Bacillus ulceris serpentis ful-

minantis 46 Bakte riologie 42 (Bericht) Bakterium coli 46 Barbitursäurevergiftung 77 Bellsches

Phänomen 118 Bilharzia 63 Blastomykose 59 Bleivergiftung 76 Blennorrhoea neonatorum,

Virus der - 54 Blindenwesen 92 Blindheit, Prophylaxe der - in

Rumänien 192 (Bb.) Blitzschlag 84 Blutdruck 106 Blutgruppen 73 Blutung, expulsive -- bei

Glau-

kom 353 Botulismus 79 Brucella 47

C Cephalosporium 59 Chemie, physiologische und physi-

kalische - 96 (Bericht) Chin in, Behandlüng des Trachoms

mit - 243 Chininvergiftung 77 Chorioidea, atypisches Kolobom 
der $\cdot-248$

Ossifikation der - 122

Sarkom der - mit negativem Durchleuchtungsbef und 118

Christian-Schüllersche Krankheit 252

Conjunctiva, allergische Erkran-kungen der -- 67

- nichtexulzerierte Sklerose der - an der Übergangsfalte des Un-terlides, der

halbmondförmigen Falte und des Augapfels 300

-. symmetrische Tumoren der -179

Cornea 232 (Bericht)

- $\quad$ degenerative Erkrankungen der

$-230$

-· allergische Erkrankungen der -68

D

Diabetes mellitus, Einfluß des

- $\quad$ auf die Refraktion 169

Sachregister zu Band 92.

375

Diabetes, Rückbildungen von

Linsentrübungen bei --178 Diathermie, Veränderungen der

Iris durch - 348 Dimethylsulfat, Augenschädi-

gung durch - 78 Dinitrophenol 74 Diphtherie, Augenkomplikatio-

nen der - 336 Diphtheriebazillen 45 Druck, intraokularer no Diplobazillen 45

- des anderen Auges bei Glaukom

353 Dysenteriebazillen 55

E Echinokokkus 63 Einschlußblennorrhoe, Virus

der - 53 Elektrische Unfälle 84 Elephantiastische Lidverän-

derungen 1 Elliotsche Operation 365 Epithelzyste, intrasklerale 275 Explosion, Verletzung durch

84

F Farbensinnprüfung 122 Febris exanthematica, Virus

der -56 Fehlanlagen am Locus minoris

resistentiae 266 Filaria 62

Flavin in der Linse 99, 102 Flüssigkeitswechsel 105 Follikulin, Vermehrung des -

im Harn bei Glaukom 355 Formaldehyd, Augenschädigung

durch - 76 Fremdkörper 84 Fukalasche Operation 169

G

Ganglion sphenopalatinum

und Auge 269 Gehirntumoren 267, 268 Gehirnschädigung, Sehätzung der Erwerbsfähigkeit bei dauern-der traumatischer - 371 Gewerbevergiftungen 79 Glaskörper, Çjuellungsdruck des

$-355$

- $\quad$ und Netzhautabhebung 115Glaukom 350 (Bericht)

- doppelseitiges - - bei Leontiasisossea 221

Glioma retinae, Röntgenbe-

handlung des - 268 Glykokolsaures Natron bei

Glaukom 360 
Goldimprägnation der Horn-

haut 242 Gonokokkus 45

- $\quad$ Allergie gegen - 69 Gravidan 360

Grippe, grippöse Hämorrhagien am Augenhintergrund 207

Grönblad-Strandbergsches Syndrom 371

Grubenbildung in Conjunctiva und Episklera 245

$\mathrm{H}$

Hemeralopie, mit Augenhinter-grundsbefund 183

Hepatolentikuläre Degeneration 174

Herpes corneae 235

- $\quad$ Pathogenese des - 215Herpes zoster, Virus des - $\mathbf{0}$ 54Herpes zoster ophthalmicus, Perineuritis und Periarteriitis ciliaris bei einem frischen Fall von -- 35 Herpesvirus 54, 72 Hundezecke 64 Hyaline Entartung, familiäre des Augenhintergrundes 188 Hyperopie, transitorische Kata-

rakt bei transitorischer diabeti-

scher - 122 Hypertonie, Sehnervenbefunde

bei allgemeiner - 113 Hyphaema, Verwendung des Star-

saugers bei - 201 Hypopyon, Verwendung des Star-

saugers bei -201

I

Immunität 65 (Bericht) Infektionskrankheiten, Augen-

komplikationen der - 336 Iridenkleisis 364 Iridozyclitis, Ätiologie der - 154

- $\quad$ intraokulare Drucksteigerungbei - 356, 357

Iridosklerotomie 364 Iris 346 (Bericht) •-- Mißbildung der - 112 Iriszyste, Radiumbehandlung

der -247

$\mathrm{K}$

Kammerbucht, Morphologie der - · bei verschiedenen Formen von Glaukom 353

Kammerwasser, Chemie des -

97

- $\quad$ Entstehung des - 105Kampfgase, Augenschädigung

durch -78

376

Sachregister zu Band 92.

Kantharidin, Augenschädigung

durch - 79 Kanülen-Lanze 248 Katarakt 121 - Klinik und Erbbiologie der an-

geborenen $-322 \cdot-$ bei Neurodermie 16

transitorische bei transitorischer diabetischer Hyperopie 122

Absaugung weicher Stare und Starreste 201

Operationsaussichten bei kom-plizierter - $-\mathbf{n} 129$

Kataraktoperation 259

- $\quad$ intrakapsuläre Extraktion mit-tels der Pinzette 369, 371

Keratitis neuroparalytica 183 ,

184, 236 Keratitis parenchymatosa 233 •- experimentelle 300 Keratitis sicca 238 Keratitis superficialis und Vitamin A 125 Keratoconjunctivitis sicca 121 
und Heredität 174 Keratokonus 238 Keratoplastik 244

Verwendung des Starsaugers bei

- 201

Kobragift, Wirkung des - bei

Glaukom 361 Koch-Weeks-Bazillen 44 Konvergenzkrampf, Diagnose

des funktionellen --251 Kunst, Darstellung des Auges in

der expressionistischen - 158 Kunstseidenindustrie, Augen-

erkrankungen in der - 191

(Bb.), 240 Kurzwellenbehandlung 179

$\mathrm{L}$

Lawrence-Biedlsche Krank-

heit 265 Leishmania 61 Leontiasis ossea, doppelseitiges

Glaukom bei - 221 Leprabazillus 48 Licht und Blendung 83 Lider, elephantiastische Verände-

rungen der - 1 Linse, physiologische Chemie der

- 99

- Ossifikation der -122

- Veränderungen der - im Ultra-schallfeld 177

Linseneiweiß, Überempfindlich-

keit gegen - 68 Linsentrübungen, Rückbildun-

gen von - bei Diabetes 178

Linsenvorderkapsel, Abschilfe-rung der - - bei Glaukom 355

Luotestreaktion 70

Lymphogranulomatosis in-guinalis, Virus der - 55

Lysozym 68, 96

$\mathrm{M}$

Magnesiavergiftung 76 Malariakeratitis 237 Marimbondo 64 Membrana pupillaris perseverans, Operation der - 186 Masern, Augenkomplikationen der

- 336 Meningokokken 45 Meskalinvergiftung 79 Mikrokokkus catarrhalis 45 Milzbrandbazillus

46 Miotika, Minimalkonzentration

der --358 Mißbildungen 192 (Bb.) Myektomia muse. obi. inf. bei

Trochlearisparese 120 Myiasis 64

Mykosis fungoides 60 Myopie 168

$\mathrm{N}$

Naevus flammeus und Glaukom

357 Nagelfalzblutungen, kapilläre

bei Augenkranken 255

Naturheilverfahren bei Glaukom 358

Nervus opticus, Befunde am - - bei allgemeiner Hypertonie 113

Nervus opticus-Atrophie, Lebersche 263

--m - Lebersche - und Unfall-folgen 177

Nervus trochlearis, Myektomie des Obliquus inferior bei Parese des $\mathbf{\square}-120$

Neurodermatitis 189

Neurodermie, Katarakt bei -16

Nikotinvergiftung 77

Nitrobenzolvergiftung 79

Novokaininjektion bei Glaukom 361 
Nystagmus der Bergleute 91

0

Oguchische Krankheit 121

Onchozerkose 62

Ophthalmia sympathica, Über-empfindlichkeit bei - 69

Ophthalmoplegie, kindliche progressive - mit Veränderung des Röntgenbildes 185

Orbita, Verletzungen der -88

Sachregister zu Band 92. 377

Ossifikation der Chorioidea und

Linse 122 Osteodermopathisches Syn-

drom 1 Oxalsäure im Kammerwasser 97

$\mathrm{P}$

Paederus 64 Paraphenylendiamin 76 Parasiten 59 (Bericht) Pathologie, vegetative 96 (Ber.)

Periektomie 191 Perineuritis und Periarteriitis

ciliaris bei Herpes zoster oph-

thalmicus 35 Physiologie, vegetative 96 (Ber.) Plica semilunaris, nichtexulze-

rierte Sklerose der - 300 Pneumokokken 44 Prellung 83 Presbyopie, Leistungsfähigkeit

des pr. Auges bei wechselnder

Pupillenweite 250 Pseudoisochromatische Ta-

feln 122 Pseudotumor, Histologie dessub-

makularen senilen - 306 Pterygium 242 Pupillotonie 252

$\mathrm{R}$

Radio graphie en Ophthalmo-

logie 373 (Bb.) Radiumschädigung des Auges

241 Refraktion bei den Eingeborenen

in Gabon 121Refraktionsanomalien 168

(Bericht) Retikuloendotheliale Zellen

in der Hornhaut 232 Retina, chemische Untersuchung

der - 101 - scheibenförmige Entartung der

mitte 306

Retinitis unbekannten Ursprungs

192 (Bb.) Retinitis circinata 180 Rezepttaschenbuch 192 (Bb.) Röntgenbehandlung bei Glau-

kom 361 Rosace a, Augenerkrankungen bei

- 252 Rotzbazillus 46

S Schädelverletzungen 89 Scharlach, Augenkomplikationen

des - 336 Schlangengift, Augenschädigung

durch $-\square 79$

Schwefeldioxyd, Augenschädigung durch - 76

Schwefelwasserstoff als Ur-sache der Augenerkrankungen in der Viskose-Kunstseidenin-dustrie 191 (Bb.)

Schweinecholera, Virus der 54

Sehschärfe 168

- $\quad$ Verhütung der Simulation beiBestimmung der $\mathbf{a}-119$

Sehschwäche 93 Sehtäuschungen bei der Korrek-

tion von Anisometropie 119 Simulation, Verhütung der -

bei der Sehschärfebestimmung 
bei Amblyopen 119 Skiaskopie 117 Sklera 244 (Bericht)

- $\quad$ senile Entartung der --179Soorpilz 60

Soziale Medizin 90 Spirochaeta pallida 49 Sporotrichose 60 Staphylokokken 43

- $\quad$ Immunisierung gegen - 69Stauungspapille 176Streifenbildungen in der Hornhaut 242

Streptokokken 44

- $\quad$ Immunisierung gegen 69Streptothrix 60Synopsien, akustisch-chromati-

sche 258 Syphilis, Allergie bei - 70 Syphilom der Sklera 245

$\mathrm{T}$

Tachistoskop 172 Tätowage mit Goldchlorid 243 Tetanusbazillen 46 Thelazia 63

Thrombangitis obliterans 182 Tollwut, Virus der - -56 Totische Operation 260 Trachom 127

(Bb.), 234

Erreger des 49

Chininbehandlung des - 243 Tränengas 186, 241 Tränenröhrchen, überzählige

Bildungen von abnorm gelager-ten -27

Trichiasis, marginoplastisches Operationsverfahren bei - 371

Trichinose 63

Trichophyton asteroides 60

Trypanosoma 62

Tuberkelbazillen 47

Tuberkulose, Immunisierung gegen $-\cdot 70$

--· tub. Augenerkrankungen als Be-rufskrankheiten 92

378

Sachregister zu Band 92.

Tularämie 47, 185, 249 Turn or en und Unfall 90

- $\quad$ Röntgenbestrahlung intraoku-lärer -263

$\mathrm{U}$

Ulcus serpens 234 Ultraschallfeld 177 Uvea, Allergie der - 69

$\mathrm{V}$

Vegetative Physiologie und

Pathologie 96 (Bericht) Verätzungen 82, 186, 240 Vergiftungen 74 (Bericht) Verletzungen 81

(Bericht), 240 Versicherungswesen 90 Vitamine, lokale Verwendung von Vit.-A-haltigen Salben 256

- $\quad$ A-Vit. und Hornhaut-Netzhaut-leiden 124, 125

Vitamine, C, Gehalt des Kam-merwassers an -98

W

Wimpernfärbemittel, Schädi-gungen durch - 75, 76, 127

$\mathrm{X}$

Xerophthalmie, durch fettfreie Ernährung erzeugte --124

Z 